\title{
Validez de constructo, confiabilidad y punto de corte de la Prueba de Síntomas Subjetivos de Fatiga en trabajadores mexicanos
}

\author{
Tonatiuh Barrientos-Gutiérrez, M en C en S en el T, (1) Susana Martínez-Alcántara, M en Med SoC,, ${ }^{(1)}$ \\ Ignacio Méndez-Ramírez, Dr en Est. ${ }^{(2)}$
}

\section{Barrientos-Gutiérrez T, Martínez-Alcántara S,} Méndez-Ramírez I. Validez de constructo, confiabilidad y punto de corte de la Prueba de Síntomas Subjetivos de Fatiga en trabajadores mexicanos. Salud Publica Mex 2004;46:516-523. El texto completo en inglés de este artículo está disponible en: http://www.insp.mx/salud/46/eng

\section{Resumen}

Objetivo. Evaluar la validez de constructo, la confiabilidad interna y establecer un punto de corte para la Prueba de Síntomas Subjetivos de Fatiga. Material y métodos. Esta prueba fue desarrollada en Japón para medir fatiga en población trabajadora, cuenta con 30 reactivos divididos en tres esferas. La validez de constructo fue explorada por diferencias entre grupos, utilizando una población de 1399 trabajadores de ocho empresas de la Ciudad de México que fueron estudiadas en 1999. Se utilizó como red nomológica el modelo de control-demanda de Theorell y Karasek derivando con ello hipótesis de trabajo. Los trabajado res fueron clasificados en nueve grupos dependiendo de su nivel de exigencia-control, y las diferencias en la puntuación de fatiga se derivaron mediante análisis de covarianza (AN C O VA). La confiabilidad interna se obtuvo a través del alfa de Cronbach. El punto de corte se definió a partir de la relación lineal esperada entre las exigencias laborales y la fatiga. Resultados. La distribución de la puntuación en la Prueba de Síntomas Subjetivos de Fatiga resultó similar a la esperada, que aumenta conforme se incrementa la exigencia o disminuye el control. Las diferencias entre grupos fueron estadísticamente significativas. El alfa de C ronbach para la prueba en general fue de 0.89 , mientras que el valor por esfera fue siempre superior a 0.70 . Los puntos de corte con una correlación más estrecha fueron 6 y 7 respuestas afirmativas ( $r=0.9456$ y 0.9479 , respectivamente). Conclusio-

\author{
Barrientos-Gutiérrez T, Martínez-Alcántara S, \\ Méndez-Ramírez I. \\ Construct validity, reliability, and cutoff point \\ of the Subjective Symptoms of Fatigue Test \\ in Mexican workers. \\ Salud Publica Mex 2004;46:516-523. \\ The English version of this paper \\ is available at: http://www.insp.mx/salud/46/eng
}

\begin{abstract}
A bstract
Objective. To evaluate the construct validity and the internal reliability of the Subjective Symptoms of Fatigue Test (SSFT), as well as to establish its best cutoff point. Materials and Methods. The SSFT was developed in Japan to measure fatigue in a population of workers; the test includes 30 items in three spheres. Construct validity was explored through differences among groups, using a po pulation of 1399 workers from eight industries studied in Mexico City in 1999. We used the Theorell and Karasek's control-demand model as our nomological network, from which we derived two work hypotheses. Workers were divided into nine groups according to their level of control-demand; fatigue differences were assessed using analysis of covariance. The internal reliability level was tested with Cronbach's alpha. The cutoff point was obtained by means of the expected linear relation between the labor demands and fatigue. Results The distribution of SSFT values was similar to the expected values, increasing proportionally with increasing demands or with decreasing control.The differences betw een groups were statistically significant. C ronbach's alpha was 0.89 , while the value per sphere was always above 0.70 . The cut-off points most closely correlated were 6 and 7 affirmative answers. Conclusions. The observed score obtained in the distinct groups was as expected, in agreement with the study hypotheses. The internal reliability of the test was adequate. The proposed cutoff point for the general population was
\end{abstract}

(1) Universidad Autónoma Metropolitana-X ochimilco. México, DF, México.

(2) Instituto de Investigaciones en Matemáticas A plicadas a Sistemas, Universidad N acional Autónoma de México. México, DF, México.

Fecha de recibido: 19 de agosto de 2003 - Fecha de aprobado: 13 de agosto de 2004 Solicitud de sobretiros: Dr.Tonatiuh Barrientos Gutiérrez, Paseo de las G alias, 31-403, Fraccionamiento Lomas Estrella, Delegación Iztapalapa, 09890 México, DF, México.

Correo electrónico: tonatiuhb@ hotmail.com 
nes La puntuación observada en los distintos grupos se comporta de la forma esperada, cumpliendo así las hipótesis de desempeño planteadas. La confiabilidad interna para la prueba en general es adecuada. El punto de corte propuesto para población general es de siete respuestas afirmativas. El texto completo en inglés de este artículo está disponible en: http://www.insp.mx/salud/46/eng

Palabras clave: cuestionario; validez; reproducibilidad de resultados; salud ocupacio nal; fatiga; M éxico seven affirmative responses. The English version of this paper is available at: http://www.insp.mx/salud/46/eng

Key words: questionnaire; validity; reproducibility of results; occupational health; fatigue; Mexico
$\mathrm{L}$ a fatiga es una sensación compleja, integrada por síntomas físicos y psíquicos, que ubica a quien la percibe en un continuo que va desde sentirse bien hasta estar exhausto; constituye un sistema de evaluación de la integridad del individuo y un factor determinante en la disposición para realizar alguna tarea. En el campo de la salud en el trabajo la ubicación del estrés y la fatiga como mediadores psicofisiológicos entre el proceso de trabajo y diversos daños en la salud de los trabajadores ha abierto un conjunto de nuevas opciones para el diagnóstico y la prevención. ${ }^{1}$ Desde este precepto el estudio de ambas condiciones se transforma en un indicador confiable de los efectos inmediatos y potenciales que el proceso de trabajo tiene sobre la corporeidad y el psiquismo del trabajador.

Por estos aspectos la evaluación de la fatiga ha sido central para la salud en el trabajo, obligando al desarrollo constante de instrumentos que, a través de indicadores fisiológicos o de pruebas subjetivas, permitan estimar su intensidad y características. Una de ellas es la Prueba de Síntomas Subjetivos de Fatiga (PSSF), que fue desarrollada por el Comité para la Investigación de la Fatiga Industrial, de la Asociación de Salud Industrial del Japón, en 1954. Este instrumento consta de 30 reactivos que exploran la presencia de síntomas que originalmente fueron clasificados en tres grupos: físicos, mentales y neurosensoriales. ${ }^{2}$

Saito, Kogi y Kashigawi, en 1970 sometieron a validez factorial el instrumento obteniendo tres factores: somnolencia y pesadez, proyección de malestar físico y dificultad para concentrarse. ${ }^{2}$ Yoshitake en 1978 relacionó el primer factor con trabajo indiferenciado, el segundo con trabajo físico y el tercero con trabajo mental; adicionalmente propuso la calificación de la prueba a través del porcentaje de respuestas afirmativas. ${ }^{3}$

Almirall en 1982 validó por criterio la PSSF en población cubana. ${ }^{4} \mathrm{Su}$ aplicación en Cuba, México, Venezuela y Brasil ha incluido periodistas, controladores de tránsito aéreo, trabajadores siderúrgicos y refresqueros. $^{4-7}$

La utilidad de la PSSF ha sido constatada en el estudio integral de pacientes con diversas patologías. Su uso en el ámbito de la salud pública no sólo se ha restringido a los estudios epidemiológicos realizados con trabajadores, también han sido estudiados pacientes con cáncer, síndrome de fatiga crónica, síndrome de inmunodeficiencia adquirida y mujeres en periodo de posparto, por citar algunos ejemplos., 8

La validación en trabajadores mexicanos de la PSSF pondrá a disposición de la comunidad académica y científica un instrumento de rápida aplicación y fácil manejo para la evaluación del nivel de fatiga presente en población trabajadora de nuestro país. Estas características la hacen ideal para la aplicación masiva en los centros de trabajo, facilitando la realización de estudios de corte epidemiológico.

Los objetivos de este estudio son: a) evaluar la validez de constructo de la PSSF en trabajadores mexicanos a través de las diferencias entre grupos; b) explorar la confiabilidad interna, y c) determinar un punto de corte que permita dicotomizar el resultado.

\section{Red nomológica de fatiga}

Para llevar a cabo el estudio de la validez de constructo por diferencias entre grupos de la PSSF acudimos al desarrollo teórico efectuado por Cronbach, ${ }^{10}$ quien establece que dicha validez descansa en la claridad alcanzada en el desarrollo del concepto en estudio (constructo) y de su red nomológica. La red nomológica es el conjunto de leyes o condiciones que determinan el comportamiento del constructo. Una vez definida ésta es posible derivar hipótesis comprobables empíricamente que, en el caso de las diferencias entre grupos, son predicciones acerca del desempeño en la prueba que mostrarán grupos con características distintas. 
La red nomológica de la fatiga laboral ha sido estudiada de forma aislada o a través de modelos dinámicos, como las propuestas de Grandjean, Stellman y Daum, o Theorell y Karasek. ${ }^{11-13}$ A partir de estas tres propuestas construimos para este estudio un modelo unificado, que nos permitiera derivar hipótesis (figura 1).

Los estudios de fatiga laboral han centrado su atención en distintas condiciones que casi en su totalidad derivan del proceso de trabajo. Este proceso es fundamental, ya que de sus características dependen el tipo y cantidad de condiciones con las que interactúa el trabajador. Como se muestra en el esquema 1, la generación de fatiga a partir de estas condiciones no sólo obedece a la cualidad y cantidad de éstas, sino también y, de forma muy importante, de la posibilidad que el trabajador tenga para controlarlas. Aquellas condiciones que sobrepasen la posibilidad de control por parte del trabajador se transformarán en riesgos y en estresores, exigencias o demandas psicológicas, ${ }^{*}$ es decir en elementos que potencialmente pueden generar una respuesta de estrés en el trabajador (entendiendo estrés en sentido amplio). El trabajador se enfrenta en este momento a un medio que puede resultar hostil. Dependiendo de su experiencia e interpretación, sobrellevará aquellas cosas que no le afecten y responderá a aquellas que le signifiquen una molestia, un reto o un conflicto, lo que se transformará en estrés vivido. En este momento y dependiendo de las condiciones particulares del trabajador y de la restricción del medio circundante se echará mano de un último recurso para disminuir el contacto con el estrés: las estrategias de resistencia cotidiana. Estas fungen como controles extraordinarios que irrumpirán en el proceso de trabajo, estableciendo distancia entre éste

\footnotetext{
* Los diversos requerimientos que el medio laboral impone a los trabajadores y que pueden ocasionar una respuesta de estrés han recibido diferentes denominaciones, tales como factores psicosociales, estresores laborales, demandas psicológicas o exigencias laborales. En esencia, en todos estos conceptos está implícita la valoración que los individuos realizan sobre determinadas condiciones del medio circundante y su capacidad de respuesta. Cuando el sujeto se percibe con capacidad de respuesta adecuada al requerimiento ambiental, la consecuencia final es de aprendizaje y crecimiento. Sin embargo, cuando el sujeto valora que su capacidad de respuesta es limitada, el estrés adquiere un carácter negativo y, de mantenerse por mucho tiempo, tenderá hacia la cronicidad, con consecuencias negativas para la salud. En este trabajo se asumen como sinónimos el concepto de demanda, propuesto por Theorell y Karasek ${ }^{13}$ y el de exigencia laboral, propuesto por Noriega ${ }^{14}$, ya que ambos se refieren a condiciones laborales objetivas con capacidad de desencadenar respuestas como las descritas anteriormente.
}

y el trabajador, y alejándole de la situación estresante a través de mecanismos que abren espacios de descanso o relajamiento.

De acuerdo con Stellman y Daum la fatiga es producida por la acumulación de diversos tipos de estrés, es decir, la fatiga es la impronta que el estrés deja en el trabajador una vez que la situación crítica ha pasado, es el desequilibrio energético al que el trabajador se expone cuando el proceso lo expolia. ${ }^{12}$ Así, tanto el estrés como la fatiga constituyen mediadores psicofisiológicos entre las condiciones laborales y los daños a la salud, sin que su presencia caiga necesariamente en el terreno de la patología. ${ }^{1}$

La relación entre el nivel de fatiga y la valoración que el sujeto hace de su entorno está íntimamente relacionada, de manera que al aumentar el nivel de fatiga se modificará la interpretación que el sujeto haga de los estresores externos, quien a su vez pondrá en acción más estrategias de resistencia como una forma de protección.

Con la construcción de este modelo puede observarse con facilidad que en la generación de la fatiga laboral se encuentran inmiscuidos un conjunto de fenómenos y condiciones de gran complejidad. Este trabajo propone la validación de la PSSF por diferencias entre grupos, requiriendo un marco teórico a partir del cual derivar hipótesis, por lo que optamos por reducir la red nomológica a dos de sus componentes más relevantes y objetivos: las exigencias o demandas laborales y el control sobre el trabajo, a través del modelo propuesto por Theorell y Karasek. Este modelo plantea que la interacción entre las demandas psicológicas y el control en el trabajo determina el impacto sobre el trabajador; así, a mayor exigencia y menor control habrá más deterioro, mientras que conforme aumente el control o disminuya la exigencia el impacto será menor. ${ }^{13}$

El modelo fue diseñado para el estudio del estrés y condiciones derivadas de él, entre las que se encuentra el cansancio (exhaustion). En este sentido, se encontró una estrecha relación entre el nivel de cansancio y el nivel de demanda psicológica-control que puede observarse en la figura 2.

\section{Material y métodos}

\section{Población en estudio}

Se recuperaron 1399 casos de ocho bases de datos del archivo de la Maestría en Ciencias en Salud en el Trabajo. Estas bases fueron capturadas por alumnos de la maestría pertenecientes a la generación 1999-2000 con el objetivo de desarrollar la idónea comunicación de resultados para la obtención del grado. Todos los estu- 


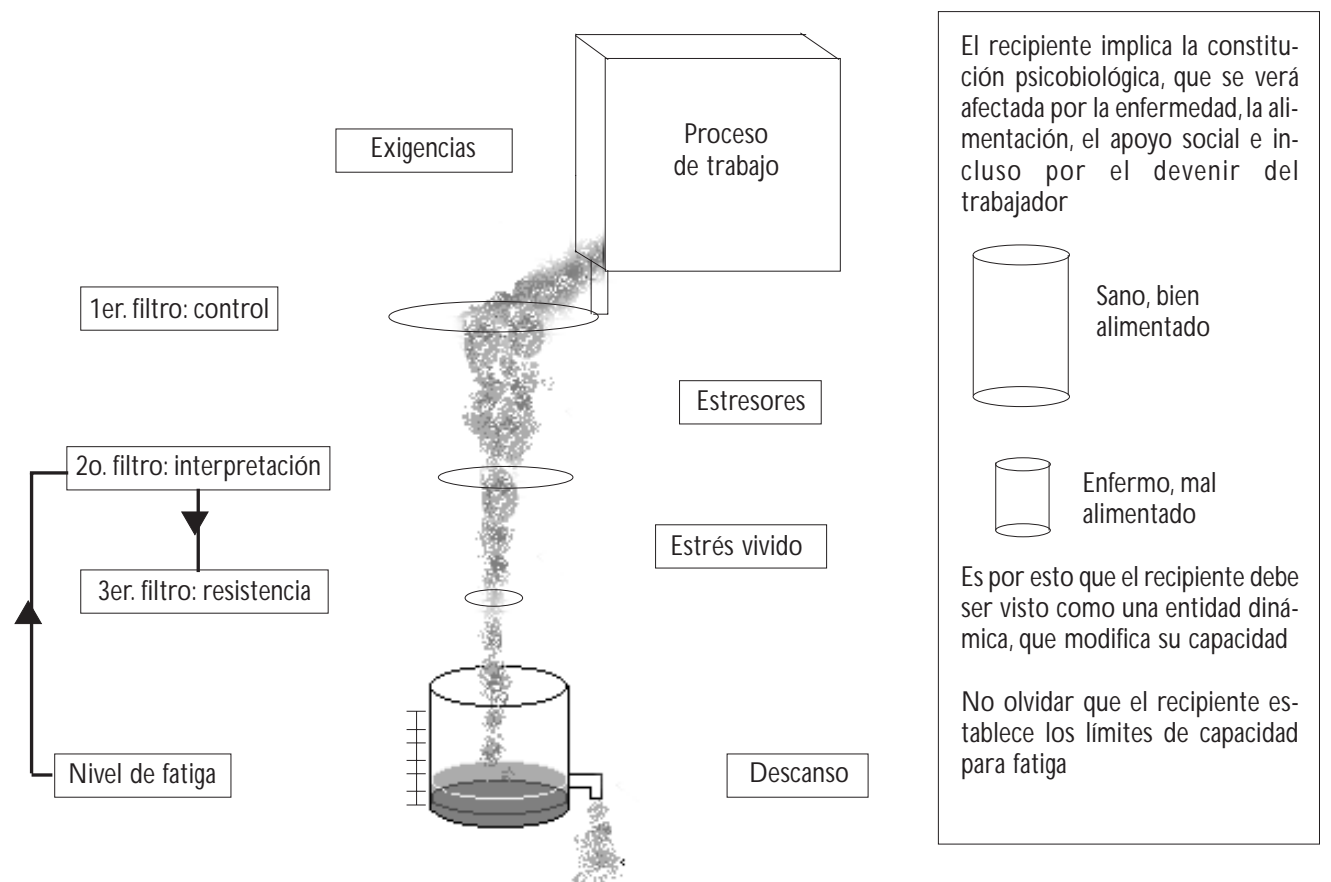

Figura 1. Determinación de la fatiga en el proceso de trabajo: modificación al modelo de Grandjean

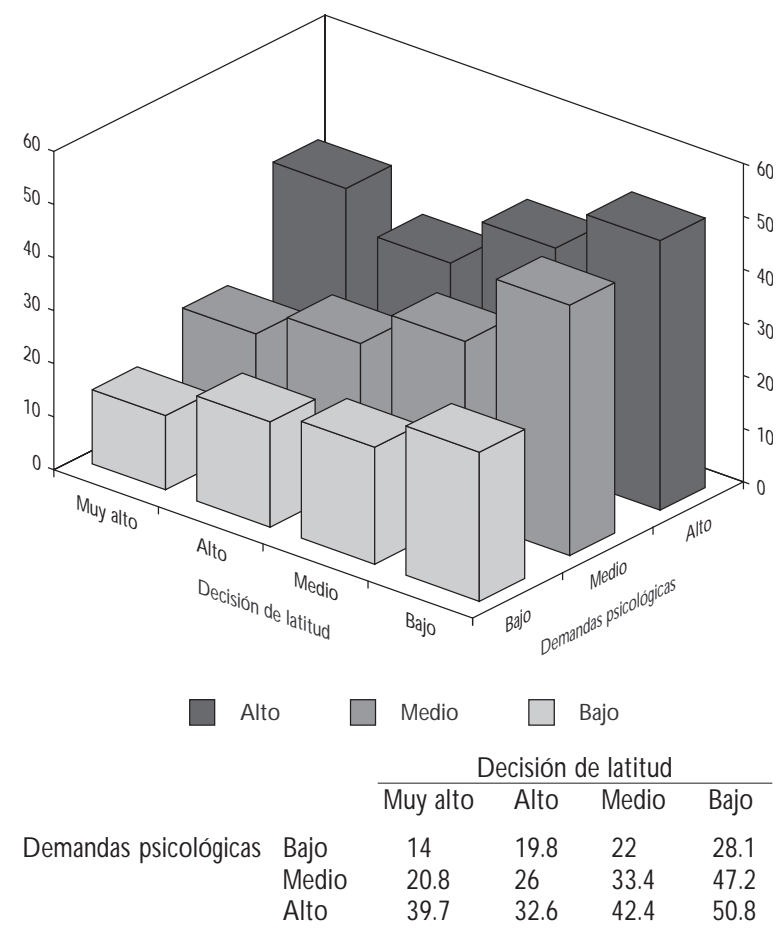

Figura 2. Cansancio en varones suecos dios utilizaron la misma metodología y el mismo sistema de captura y análisis.

Se trata de estudios transversales, observacionales, cuyo objetivo primordial era establecer la relación entre los perfiles patológicos de los trabajadores y las condiciones de trabajo. Todas las investigaciones cuentan con una descripción breve de las características de las empresas estudiadas, las áreas y puestos más importantes así como de los procesos de trabajo. El instrumento aplicado es el Programa de Evaluación y Seguimiento de la Salud Laboral (Proessal) que está constituido por la Guía para el Estudio de las Condiciones de Seguridad e Higiene en los Centros de Trabajo y la Encuesta Individual de Autollenado sobre Condiciones de Trabajo y Salud. Se recolectó información demográfica, de vida cotidiana, salud y trabajo en ocho empresas de la zona metropolitana de la Ciudad de México, pertenecientes a diferentes ramos de la industria: alimentos $(7 \%)$, construcción ( $8 \%)$, plástico $(8 \%)$, restaurantera $(9 \%)$, metalmecánica $(30 \%)$, autopartes $(14 \%)$, vestido $(17 \%)$ y editorial $(7 \%)$. En cuatro de estas empresas se incluyó a más de $90 \%$ de la población trabajadora, en las otras cuatro sólo se incluyó a la población que quiso participar en el estudio, sin aplicar ningún método que asegurara aleatoriedad. 


\section{Validez de constructo por diferencias entre grupos}

El planteamiento de las diferencias entre grupos derivó de la correlación entre exigencias y control postulada en el modelo de Theorell y Karasek. Este define una relación antagónica entre las exigencias laborales y la capacidad para controlarlas, que puede o no ser absoluta, por lo que cabe esperar que la relación se dé en términos de disminución o suavizamiento del efecto de las exigencias en función de la cantidad de control, en síntesis:

\section{Impacto o daño= Exigencia / Control}

Para construir las hipótesis sobre el comportamiento de los grupos a partir de este postulado simulamos el modelo. Definimos tres niveles de exigencia y tres de control asignándoles nombres como "bajo", "medio" y "alto". Asumiendo que "bajo" es la mitad de "medio" y un tercio de "alto" (lo cual es arbitrario), podemos sustituir estas gradaciones por números, siendo 1 para "bajo", 2 para "medio" y 3 para "alto". Llevando esto a una matriz, se produciría una correlación como la siguiente:

\begin{tabular}{|c|c|c|c|}
\hline E & Baja & Media & Alta \\
C & 1 & 2 & 3 \\
\hline $\begin{array}{r}\text { Bajo } \\
1\end{array}$ & 1 & 2 & 3 \\
\hline $\begin{array}{c}\text { Medio } \\
2\end{array}$ & 0.5 & 1 & 1.5 \\
\hline $\begin{array}{r}\text { Alto } \\
3\end{array}$ & 0.3 & 0.6 & 1 \\
\hline
\end{tabular}

Esta matriz nos permite observar que conforme aumenta la exigencia o disminuye el control aumenta el impacto; así, a un mismo nivel de exigencia el impacto se modifica dependiendo del nivel de control. De la misma manera, algunos grupos con condiciones diferentes de exigencia-control pueden mostrar un impacto similar al establecer la correlación (grupos de impacto homogéneo). Nótese que el impacto esperado para grupos como bajo-baja, medio-media y alto-alta es muy similar; algo parecido sucede entre otros grupos paralelos (baja-medio y media-alto, por un lado, y media-bajo y alta-medio, por el otro).

A partir de estas observaciones se pueden plantear tres hipótesis comprobables estadísticamente mediante análisis de covarianza:

1. Conforme el nivel de exigencia aumenta, lo hace también el nivel de fatiga, debiendo observarse di- ferencias estadísticamente significativas entre grupos con niveles de exigencia distintos.

2. Conforme el nivel de control disminuye el nivel de fatiga aumenta, por lo que deberán observarse diferencias estadísticamente significativas entre grupos con niveles de control distintos.

3. Los resultados deben mostrar una puntuación similar en los grupos de impacto homogéneo, quienes no mostrarán diferencias estadísticamente significativas.

Establecidas las hipótesis, se procedió a la construcción de los grupos de acuerdo con el nivel de exigencia-control. A partir de las bases recuperadas se seleccionaron las variables correspondientes a 18 exigencias (demandas), cinco elementos de control* y la PSSF. Para cada caso se realizó la sumatoria de exigencias, control y la PSSF.

Una vez obtenidas las sumatorias se clasificó a los trabajadores dependiendo del número de exigencias y elementos de control que referían; se observaron muy pocos casos con más de 14 exigencias, por lo que se procedió a su exclusión del estudio. Los casos restantes (1 371) fueron agrupados de acuerdo con el nivel de exigencia y nivel de control, y el control se dividió en tres niveles: "bajo", si la sumatoria de respuestas afirmativas se encontraba entre 0 y 1 , "medio", si ésta se encontraba entre 2 y 3 , y "alto", si el valor se situaba entre 4 y 5 . Las exigencias se agruparon en tres niveles, "baja", si las respuestas afirmativas se encontraban entre 0 y 3, "media", entre 4 y 7, y "alta", en 8 y más. De esta forma todos los casos quedaban clasificados en uno de nueve posibles grupos.

Finalmente, se calculó la media de respuestas afirmativas para cada grupo y se sometió la puntuación de la PSSF a análisis de covarianza utilizando SPSS 10.0. El control de las variables confusoras con análisis de covarianza (ANCOVA) incluyó edad, estado civil, sexo, tener otro trabajo, antigüedad en la empresa y antigüedad en el puesto.

\footnotetext{
* Exigencias: jornada mayor de 48 horas a la semana, trabajo pendiente en horas o días de descanso, rotación de turnos, trabajo nocturno, atención sostenida, mucha concentración para evitar accidentes, tarea minuciosa, trabajo repetitivo, cubrir cuota de producción, realizar trabajo a destajo, estar bajo supervisión estricta, cumplir un estricto control de calidad, estar sin comunicación con los compañeros, trabajar en un espacio reducido, estar fijo en su lugar de trabajo, recibir órdenes confusas de los superiores, realizar esfuerzo físico muy pesado, adoptar posiciones incómodas. Control del trabajo: desarrollar habilidades y destrezas, desarrollar creatividad e iniciativa, aprendizaje de nuevos conocimientos, decidir cómo realizar su trabajo, fijar el ritmo del trabajo.
} 


\section{Determinación del punto de corte}

La calificación propuesta por Yoshitake para la PSSF está basada en el porcentaje de respuestas afirmativas en la prueba. Esto puede resultar útil, pero en algunos casos se requiere la clasificación de los casos en fatigados y no fatigados.

Existen diversos procedimientos para establecer el punto de corte, pero exigen que se cuente con un criterio que pueda servir como estándar de oro. Dado que en nuestro país no existe una prueba capaz de servir para este propósito, se planteó una vía alternativa para definir un punto.

Asumiendo que existe una relación lineal entre la cantidad de exigencias a que está expuesto un trabajador y el grado de fatiga que experimenta, se planteó que el punto de corte más adecuado sería aquel que representara de manera más estrecha esta relación. Para ello se agrupó a los trabajadores dependiendo del total de exigencias que referían, de lo que se obtuvieron 12 grupos. Cada grupo fue dividido a su vez en fatigados y no fatigados, y se movió el punto de corte de 1 a 10 respuestas. Finalmente, se aplicó correlación de Spearman al porcentaje de fatigados para cada grupo de exigencia.

\section{Confiabilidad interna}

Para obtener la confiabilidad interna de la prueba se aplicó el alfa de Cronbach utilizando SPSS 10.0.

\section{Resultados}

La distribución por género mostró que $60 \%$ de la población fueron hombres y $40 \%$ mujeres. La media de edad se situó en 32.6 años, con desviación estándar de 9.88. El 60\% de los encuestados tiene pareja, 35\% son solteros, $3 \%$ divorciados y $2 \%$ viudos.

\section{Validez de constructo por diferencias entre grupos}

Se graficó la media de respuestas afirmativas en la PSSF para cada grupo, y se presentó una distribución similar a la encontrada por Theorell y Karasek (figura 3). En el gráfico puede observarse la tendencia general del nivel de fatiga a incrementarse conforme el control disminuye y la exigencia aumenta, lo cual cumple dos de las condiciones de la red nomológica. Al analizar el comportamiento de la puntuación de fatiga en relación con el nivel de exigencia puede observarse que en todos los grupos, conforme aumenta la exigencia aumenta el número de respuestas afirmativas en la PSSF. La

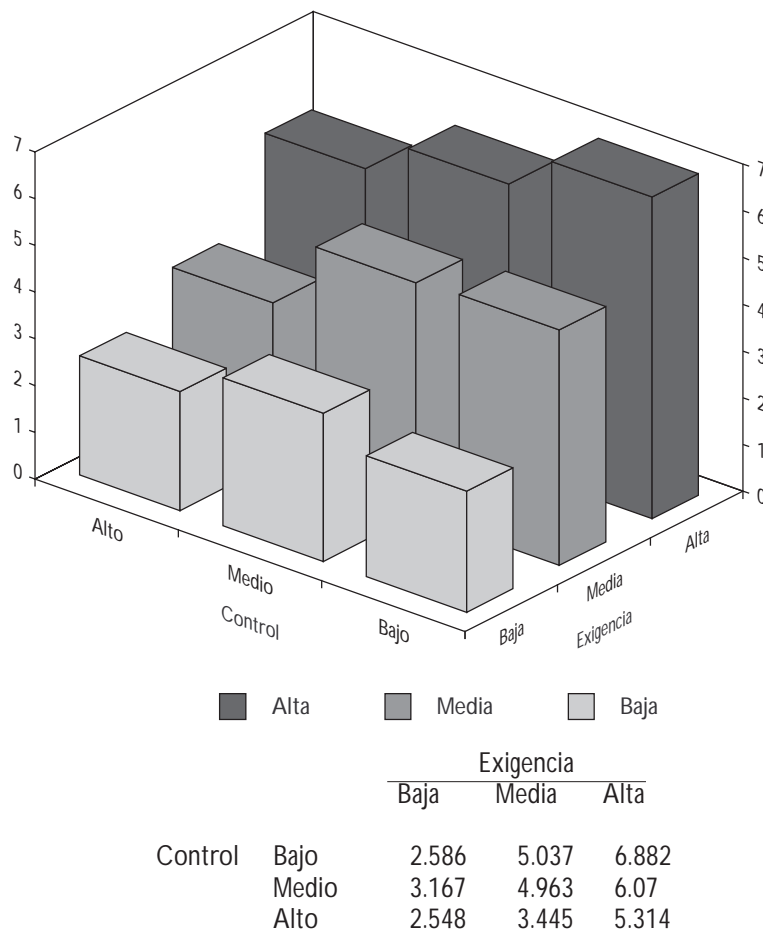

Figura 3. Media de Respuestas afirmativas a la Prueba de Síntomas Subjetivos de Fatiga, de acuerdo con EL NIVEL DE EXIGENCIA-CONTROL (CONTROLANDO SEXO). MÉxICO, DF, 1999

relación entre fatiga y control presenta un comportamiento adecuado, donde la fatiga disminuye conforme el control aumenta, especialmente a niveles altos de exigencia, ya que cuando la exigencia es baja o media el control no produce una disminución tan nítida (figura 3).

Una vez observado el comportamiento de la puntuación de la prueba entre los grupos se procedió a establecer si las diferencias eran estadísticamente significativas, para lo cual los datos se sometieron a ANCOVA; en éste se obtuvieron diferencias estadísticamente significativas en exigencia y control para la puntuación en la PSSF, tanto en el modelo simple como controlando el sexo.

El control de variables confusoras por ANCOVA demostró que el sexo tiene efecto sobre la interacción entre exigencia y control, razón por la que fue incluido en el modelo. De forma independiente se analizó el efecto de esta variable sobre la exigencia y el control colocándola como factor fijo y generando un ANCOVA con los tres factores. Tras eliminar las interacciones no significativas se concluyó que el efecto se observa en la interacción entre el sexo y el nivel de exigencia. Al 


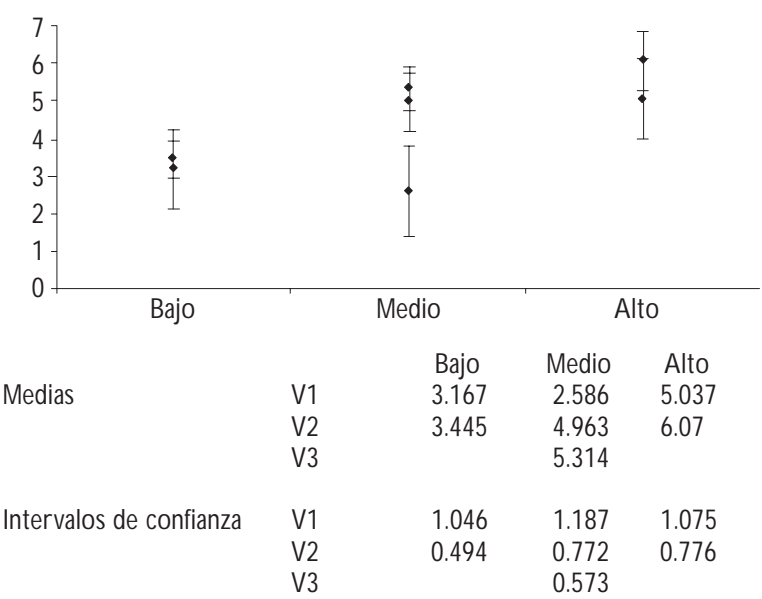

* Bajo: Baja/Medio, Media/Alto; Medio: Baja/Bajo, Media/Medio,Alta/Alto; Alto: Media/Bajo,Alta/Medio

Figura 4. Medias e intervalos de confianza para la puntuación en la Prueba de Síntomas Subjetivos de Fatiga, para grupos de impacto homogéneo.* México, DF, 1999

generar el gráfico de perfil para la interacción ésta se evidencia en el nivel de exigencia baja, donde las líneas pierden el paralelismo observado a nivel medio y alto. En el caso del control no se observó interacción con el sexo.

Para observar el comportamiento de los grupos de impacto homogéneo se generó la figura 4 utilizando las medias ajustadas y sus respectivos intervalos de confianza. No se encontraron diferencias significativas en los grupos homogéneos bajo y alto. En el conjunto correspondiente a impacto homogéneo medio el grupo de exigencia baja y control bajo tiende a puntuar significativamente por debajo de los grupos de exigencia media/control medio y exigencia alta/control alto, siendo las diferencias entre estos dos últimos no significativas. De esta forma la hipótesis de equivalencia en el impacto para los grupos homogéneos se apoya parcialmente.

\section{Determinación del punto de corte}

El punto de corte con una correlación más estrecha se ubica en dos respuestas positivas a los reactivos de la prueba $(r=0.951)$, pero con esta clasificación una proporción muy grande de la población quedaría clasificada como fatigada, por lo que consideramos que este punto de corte es poco exigente. El siguiente punto de correlación alto se observa en seis $(r=0.9456)$ y siete $(r=0.9479)$ respuestas, siendo esta última ligeramente más cercana a uno.

\section{Confiabilidad Interna}

El nivel de confiabilidad para la prueba en su conjunto presentó un alfa de Cronbach de 0.8906. Los niveles de confiabilidad interna para cada dimensión de la prueba fueron calculados, obteniéndose niveles aceptables para las tres esferas: Somnolencia y pesadez (0.8214), Dificultad para concentrarse (0.7356) y Proyección de malestar físico (0.7201).

\section{Discusión}

La fatiga es el centro de gravedad de este trabajo, su conceptualización y sus determinantes sustentan el esfuerzo de validez. Sin duda, existen cuestionamientos fuertes en lo relativo al concepto de fatiga: qué puede ser considerado como tal y qué no, qué particularidades muestra la fatiga laboral en relación con otros tipos de fatiga y, particularmente, qué aspectos de la vida determinan su presencia. Con las salvedades que implica el continuo desarrollo teórico, consideramos que el modelo aquí presentado resume las posturas más representativas y aceptadas en el campo de la salud en el trabajo, lo que constituye una síntesis con buenas posibilidades de aplicación en futuras investigaciones.

Puesto que la validez de constructo depende directamente de la fortaleza de su red nomológica, es preciso que exista consenso en la capacidad explicativa de ésta para pretender la validación. En nuestro caso no existe un consenso general acerca de las leyes o fenómenos que determinan el nivel de fatiga de una población; al respecto, pueden encontrarse distintas posiciones, lo que abre el espacio a la construcción de distintas redes nomológicas y a la discusión de la bondad de cada una. La red utilizada en este trabajo se construyó alrededor de un híbrido entre el modelo de control-demanda de Theorell y Karasek, y la propuesta de exigencias de Noriega, ${ }_{1}^{14}$ ya que consideramos que ambas posiciones han demostrado teórica y empíricamente su amplia capacidad explicativa en el ámbito de la salud en el trabajo, y son bases sólidas de las cuales derivamos las hipótesis presentadas.

Atendiendo a las hipótesis derivadas de la red nomológica planteada y al apego que mostraron los datos con la tendencia esperada, podemos afirmar que la prueba ha mostrado validez de constructo en cuanto a diferencias entre grupos. Sin embargo, no debe olvidarse la advertencia hecha por Cronbach acerca de la enorme dificultad para afirmar que una prueba se encuentra totalmente validada. La validez no se da en un solo sentido y requiere de una construcción paulatina; de recolectar datos y pruebas que confirmen la adecuación de la prueba para el fin que fue creada e incluso después de este largo proceso aún quedarán 
dudas acerca de si se ha alcanzado la validez de forma suficiente.

A pesar de constituir poblaciones diferentes creemos que el estudio de validez factorial conducido por Saito, Kogi y Kashigawi define con suficiente solidez los tres factores constitutivos de la prueba, lo que constituye una de las razones por las que decidimos validar la prueba por diferencias entre grupos. En los últimos tiempos se ha preferido el acercamiento utilizando la validez factorial, pero ésta es sólo una forma, pues están a disposición otras opciones que pueden ser aplicadas con buenos resultados. La validez por diferencias entre grupos mantiene como condición necesaria un apego constante a los aspectos teóricos del constructo y una lógica similar a la requerida en un estudio de tipo epidemiológico, resultando de fácil aplicación y comprensión en nuestro campo.

$\mathrm{Al}$ incluir trabajadores de ocho empresas distintas pretendimos alcanzar la mayor representatividad posible con los medios a nuestro alcance, manejando los datos de forma indiferenciada, con el objetivo de probar la adaptabilidad de la prueba a distintos ambientes y condiciones laborales.

Las muestras utilizadas en la investigación son en algunos casos no aleatorias, ya que la recolección dependía de la disposición de los trabajadores a colaborar en el estudio, lo que hubiese podido constituir un sesgo. Desafortunadamente, pocas de las tesis recuperaban la información acerca de la población que no fue encuestada, impidiendo así la comparación con la población participante. Partimos del supuesto de igualdad entre ambas poblaciones, pero ante nuestra incapacidad para demostrarlo asumimos que en todo caso los resultados aplican para aquellos trabajadores " $\mathrm{co}$ operadores".

La confiabilidad de la prueba presenta un nivel muy adecuado. Dependiendo de la finalidad de ésta se plantea el valor mínimo aceptable; nuestro valor de referencia, 0.80 , fue superado ampliamente en la prueba en general, aunque encontramos valores menores para dos subgrupos de reactivos.

Ante la ausencia de instrumentos validados en nuestro país que pudiesen fungir como estándar de oro, la determinación del punto de corte dependió de la presuposición de relación lineal entre la presencia de exigencias y el aumento del nivel de fatiga. Los resultados obtenidos no son contundentes, ya que se encontró una gran cercanía entre el punto de corte en seis y siete respuestas positivas. La diferencia observada en el valor de la correlación es mínima, sin embargo proponemos el punto de corte en siete reactivos, ya que consideramos preferible mantener un criterio estricto. No obstante, ante la poca contundencia de los resultados es preferible apegarse a la propuesta de
Yoshitake, utilizando la puntuación en forma de porcentaje. $^{3}$

Finalmente, consideramos que la Prueba de Síntomas Subjetivos muestra un desempeño adecuado, lo que posibilita su utilización en el campo de la salud en el trabajo. Esto no excluye la necesidad y pertinencia de realizar más esfuerzos dirigidos a aumentar su respaldo de validez.

\section{Agradecimientos}

Agradecemos a los autores de las tesis y bases de datos sobre las que este trabajo se desarrolló, alumnos de la Maestría en Ciencias en Salud en el Trabajo: Rafael Cid, Francisca Mendoza, Sandra Cabrera, Ana Reynaldos, Carlos Morales, René Valdez, Raúl Vivaldo y Maximino de la Rosa. De la misma forma, a los asesores de estas tesis, entre los que se encuentran los autores y colaboradores del programa utilizado para la evaluación realizada: Mariano Noriega, Gabriel Franco, Jorge Villegas y Guadalupe Alvear, así como Ricardo Cuellar y José Luis Torres quienes colaboraron en la asesoría de tesis.

\section{Referencias}

1 Martínez S. El estudio de la integridad mental en su relación con el proceso de trabajo. México, DF: U niversidad Autónoma MetropolitanaXochimilco; 2000; serie académicos CBS; vol. 23: 187-199.

2 Saito Y, Kogi K, Kashiwagi S. Factors underlaying subjective feelings of fatigue.J Sci Lab 1970; 46(4): 205-224.

3 Yoshitake $H$.Three characteristic patterns of subjective fatigue symptoms. Ergonomics 1978; 21 (3):231-233.

4 A Imirall P, Seyes M. Relación entre índices subjetivos y objetivos de fatiga.Validación de una prueba. Rev Cub Hig Epid 1982; 20:239-248. 5 Escalona E, D e U rosa E, González R, Romero E, Lamarca R, Jirón C et al. Fatiga laboral en controladores de tránsito aéreo. Rev Salud Trabajo 1996; 4(2): 99-108.

6 N oriega M, Laurell C, Martínez S, Méndez I,Villegas J. Interacción de las exigencias de trabajo en la generación de sufrimiento mental. Cad Saude Publica 2000; 16(4):1011-1019.

7 Martínez S,Tamez S, O rtiz L, Méndez I. Control del trabajo, exigencias laborales y daños a la salud en trabajadores de un diario informativo en la Ciudad de México. Rev Salud de los Trabajadores 2000; 8(1):5-22. 8 C oelho F, Sawada N.A fadiga nos pacientes com cáncer de laringe. Rev Latinoam Enfermagem 1999; 7(5):103-107.

9 Milligan R, Parks P, Kitzman H, Lenz E. Measuring women's fatigue during the postpartum period. J N urs Meas 1997; 5(1):3-16.

10 Cronbach L, Meehl P. Construct validity in psychological tests. Psychol Bull 1955; 52: 281-302.

$11 \mathrm{Grandjean} \mathrm{E.} \mathrm{Fatigue} \mathrm{in} \mathrm{industry.} \mathrm{Brit} \mathrm{J} \mathrm{Ind} \mathrm{Med} \mathrm{1979;} \mathrm{36:} \mathrm{175-186.}$

12 Stellman J, D aum S. El trabajo es peligroso para la salud. México, DF: Siglo XXI; 1986: 89-104.

13 Karasek R, Theorell T. Healthy work: Stress, productivity, and the reconstruction of working life. N ueva York: Basic Books Inc; 1990. $14 \mathrm{~N}$ oriega, M. El trabajo, sus riesgos y la salud. En defensa de la salud en el trabajo. México, DF: SITUAM; 1989: 5-12. 